\title{
Quantitative Amplitude- and Phase- Contrast Plasmonic Microscopy with High Spatial Resolution
}

Yuting Yang, Chunhui Zhai, Ab Lateef Khan, Hui Yu

Submitted date: 31/05/2019 - Posted date: 03/06/2019

Licence: CC BY-NC-ND 4.0

Citation information: Yang, Yuting; Zhai, Chunhui; Khan, Ab Lateef; Yu, Hui (2019): Quantitative Amplitudeand Phase- Contrast Plasmonic Microscopy with High Spatial Resolution. ChemRxiv. Preprint.

Plasmonic microscopy is a powerful tool for nanoscopic bio and chemical sample analysis due to its high sensitivity. Here, we demonstrated the quantitative amplitude- and phase- contrast imaging capabilities of plasmonic microscopy through holographical reconstructions of the interferometric plasmonic patterns. Operating interferometric plasmonic microscopy over the surface plasmon resonance angle separates twin images, and allows for accurately mapping the amplitude and phase of surface plasmon fields. The unique capabilities enable direct visualization of complex surface plasmon fields without the need for nanoscopic probes, and high-spatial-resolution imaging of nanoparticles. The proposed technology is a promising platform for nanoplasmonic study and for various sensing purposes.

File list (2)

Quantitative amplitude- and phase- contrast plasmonic mic... (1.29 MiB) view on ChemRxiv • download file 


\section{Quantitative amplitude- and phase- contrast plasmonic microscopy with high spatial resolution}

Yuting Yang, Chunhui Zhai, Ab Lateef Khan, Hui Yu*

Institute for Personalized Medicine, School of Biomedical Engineering, Shanghai Jiao

Tong University, Shanghai, 200030, China

Correspondence should be sent to: Prof. Hui Yu

Email: hui.yu@sjtu.edu.cn

$\underline{\text { Tel/Fax: }+86-21-62933948}$ 


\begin{abstract}
Plasmonic microscopy is a powerful tool for nanoscopic bio and chemical sample analysis due to its high sensitivity. Here, we demonstrated the quantitative amplitudeand phase- contrast imaging capabilities of plasmonic microscopy through holographical reconstructions of the interferometric plasmonic patterns. Operating interferometric plasmonic microscopy over the surface plasmon resonance angle separates twin images, and allows for accurately mapping the amplitude and phase of surface plasmon fields. The unique capabilities enable direct visualization of complex surface plasmon fields without the need for nanoscopic probes, and high-spatial-resolution imaging of nanoparticles. The proposed technology is a promising platform for nanoplasmonic study and for various sensing purposes.
\end{abstract}




\section{Introduction}

In the past decades, facilitated by the introduction of powerful nanofabrication and optical analysis techniques, the development of plasmonics has seen tremendous applications into optical trapping, imaging and biosensing(1-8). Due to the unique properties of surface plasmon, objective-based surface plasmon resonance microscopy (SPRM) allows for time-resolved wide-field imaging of nanosized objects, and has been used to study various biological samples including cells(9), viruses(10), organelles(11), DNA molecules(12) and exosomes(13). Analyzing intensities of the resulting distinct parabolic patterns in SPRM images provide valuable information such as sample sizes $(10,13)$, orientations $(14)$, or distances from the metal surface $(15)$. Meanwhile, the refractive index sensitivity of phase-contrast imaging could further the development of multiplex plasmonic sensing(16). Forming a SPRM interferometry/holography has enabled phase-sensitive imaging but at the expense of complex optics(17-19). Another disadvantage of SPRM is the spatial resolution, which is limited by the micrometers-long propagating length of SPs on metal film(20, 21). Including multiple-directions illumination could improve the spatial resolution, but complicate the optical systems and lower the imaging rate(22-24).

Interpretation of SPRM as a common-path interferometry has led to solutions in determining phase information and improving spatial resolution without the need for additional optics $(16,25,26)$. By matching the SPRM patterns of individual nanoparticle with numerical simulation results to quantify phases, Liu has shown the identification of nanoparticles comprised of different materials(16). But this approach 
is only valid for nanoparticle analysis in an inefficient 'particle-by-particle' manner. More importantly, the amplitude- and phase- SPRM images are highly dependent on the focal conditions and incident angles, which lead to inconsistent nanoparticle contrasts and patterns reported(16, 27). In our previous work, we have proposed an image processing algorithm that improves the spatial resolution of amplitude- SPRM image to near diffraction limit(25, 26). Later this strategy was adopted to improve SPRM sensitivity and resolution, and developed into the interferometric plasmonic microscopy (iPM) capable of single exosome analysis using optimized methodology(13). However, phase images have not been retrievable due to information loss in the image reconstruction.

In this work, after establishing the principle of interferometric plasmonic imaging under different focal conditions, we reconstructed high-spatial-resolution phase-contrast plasmonic images using a holographical reconstruction algorithm. Correlations between far-field phase distribution and near-field SP fields, as well as the correlations between phase images and refractive index were studied both numerically and experimentally. Since the key concept is based on the interferometric/holographic interpretation of plasmonic microscopy, we will refer to our system as iPM throughout the paper instead of SPRM.

\section{Results}

\section{Configuration and principle for interferometric plasmonic microscopy}

The experimental setup is based on the iPM system (Fig.1a) described previously(13). A planar plasmon wave, which was stimulated via the Kretschmann configuration by 
a collimated laser beam at a wavelength of $680 \mathrm{~nm}$, propagates across a smooth thin metal film coated on a glass slide. When the SPs interact with objects on the surface, leakage radiation (LR) of SPs through the metal/glass substrate occurs, resulting in an object wave $O(x, y)$ that emits directionally within a small range around SPR angles. Although various modes of light arises from the interactions, either evanescent or propagating, the metal-coated glass slide works as planar antennas to selectively sense and amplify only those evanescent components(28). It has already been shown that only the in-plane components of the SP field participate to the $\operatorname{LR}(28)$. The object wave together with the reflective wave $R(x, y)$ as the reference-arm of the holography, was then collected by a camera and forms an off-axis hologram since they are coherent(25). The recorded iPM image $|H(x, y)|^{2}$, as a hologram, is thus given by

$$
|H(x, y)|^{2}=|R(x, y)|^{2}+|O(x, y)|^{2}+R^{*}(x, y) O(x, y)+R(x, y) O^{*}(x, y)
$$

For subwavelength objects, $|O(x, y)|^{2}$ is much smaller than $|R(x, y)|^{2}$, and is ignored. The constant reference term $|R(x, y)|^{2}$, together with static background noises, can be removed with a commonly used differential approach for an adsorption process or by laterally shifting or modulating the sample stage. A typical hologram of a 40-nm Ag nanoparticle is shown in Fig 1b, giving unique interference patterns. In this work, the propagating direction of SPs is always oriented in vertical direction, unless described otherwise. Note that recording the hologram at different focal planes 
would affect the imaging sensitivity and patterns due to the wave propagation in far field(27). Fig. 2a and b showed the experimentally recorded and theoretically simulated images of 40-nm Ag nanoparticles at different imaging planes, in which the LR wave $O(r, z)$ scattered from a nanoparticle located at $r$ ' on the metallic surface was described as a cylindrical wave propagating in the z-direction.

$$
O(r, z)=\left.\alpha E_{\mathrm{sp}}\left(r^{\prime}\right) e^{-\kappa \mid r-r^{\prime}}\right|^{-i\left(k_{\mathrm{sp}} \cdot\left|r-r^{\prime}\right|+\sqrt{\left|n \cdot k_{0}\right|^{2}-\left|k_{\mathrm{sp}}\right|^{2}} z\right)},
$$

where $\alpha$ refers to the scattering coefficient of nanoparticles, $E_{\mathrm{sp}}$ is the propagating SP wave respectively, and the first exponential term describes the internal damping due to absorption in metal. Note that $\alpha$ is complex number, with the amplitude describing the scattering intensity, and the phase is related to the refractive index of nanoparticles.

\section{Retrieving amplitude and phase images by holographical reconstruction}

Since the iPM images represent common-path holograms, it is therefore possible to fully recover the amplitude- and phase- images similar to the digital holography. Multiplying the hologram in Eq.(1) by a digital copy of the planar reference wave $R(x, y)$ leaves the object wave $|R(x, y)|^{2} O(x, y)$ and its twin image $R^{2}(x, y) O^{*}(x, y)$

$$
|H(x, y)|^{2} \cdot R(x, y)=|R(x, y)|^{2} O(x, y)+R^{2}(x, y) O^{*}(x, y)
$$


Simple back-propagation of Eq.(3) would result in a focused image and a defocused image of the object wave that spatially overlap. Due to wavevector matching, the LR object waves emit only within a narrow range centered at the resonance angle $\theta_{\text {SPR }}$. The angular spectrum image of the hologram calculated by taking the two dimensional Fast Fourier Transform (2D-FFT) thus contains two ring patterns corresponding to the twin images in Eq.(1) (Fig. 1c). The wavevector of the reference wave $\left(k_{0} \cdot n \cdot \sin \theta_{\mathrm{i}}\right.$, where in $k_{0}$ is the wavevector in vacuum, $n$ is the refractive index of glass and $\theta_{\mathrm{i}}$ is the incident angle) could thus be determined experimentally from the distance between the ring center and the origin, while the SPs wavevector $\left(k_{0} \cdot n\right.$. $\sin \theta_{\mathrm{SPR}}$ ) corresponds to the radius of the ring (Fig. S1, supplementary materials). The width of the ring ( $\Delta k$, damping to $\sim 1 / \mathrm{e}$ ) is determined by the SP propagation length due to the internal damping in metal films.

In previous iPM settings with an incident angle smaller than or equal to the resonance angle, the twin images overlap, and lead to inaccurate reconstruction(26). However, at an incident angle that matches $k_{0} \cdot n \cdot \sin \theta_{\mathrm{i}}>k_{0} \cdot n \cdot \sin \theta_{\mathrm{SPR}}+\Delta k / 2$ (Fig. 1e), the twin images are well separated in the angular spectrum (Fig. 1d). Therefore, by using a spatial mask $M$ to remove one of the twin image, and backpropagating the LR waves in the angular spectrum in the opposite $z$-direction, it would allow the reconstruction at any z-planes,

$$
O(x, y, z)=\mathcal{F}^{-1}\left\{\mathcal{F}\left(k_{x}, k_{y}\right)\left\{\left|H\left(x, y, z_{0}\right)\right|^{2} \cdot R(x, y)\right\} \cdot M \cdot e^{-i \sqrt{k^{2}-k_{x}^{2}-k_{y}^{2}}\left(z-z_{0}\right)}\right\},
$$


where $\mathcal{F}$ and $\mathcal{F}^{-1}$ denotes the Fourier and inverse Fourier transforms, respectively. We note that since SPs does not propagate in the $z$-direction, the recorded hologram represents a 3D projection of the quasi-2D near field fields, and the reconstructed image does not resolve object information in the z-direction(29).

The amplitude and phase images of the object wave were reconstructed from simulated holograms, and horizontal and vertical line profiles were plotted (Fig.S2, supplementary materials). The ringing effect in the reconstructed amplitude image (Fig.S2a) and the abnormally high amplitude and degradation of phase around the scatterer location $\left(E_{\text {rec}}\right)$, as compared to the true plasmon field $\left(E_{0}\right)$ are due to the shaping by the objective pupil ( $\left.E_{0 \mathrm{dl}}\right)$ (Fig.S2c). The phase image indicates the nature of an outgoing spherical wave (Fig.S2b). Since the initial phase of the reference wave is unknown, the reconstructed phase includes a constant offset from the true phase (Fig. S2d). Fig.2c shows a stack of intensity images reconstructed from a plasmonic hologram recorded at $z=2 \mu \mathrm{m}$, which correspond well to the experimental images.

\section{Mapping plasmon/nanostructure interactions}

Directly visualizing the surface-plasmon (SP) waves may facilitate fundamental studies of interactions between plasmons and nanostructures, and lead to novel plasmonic imaging devices and systems. However, it relies on either sophisticated nanoscopic probes, i.e. near-field scanning optical microscopy (NSOM)(30-32), or complex optics and nanostructures, i.e. leakage radiation microscopy (LRM)(33-35), to map the full complex SP fields. Here, we show that the proposed methodology is superior for mapping surface plasmons in that the samples do not need to be 
positioned within the close vicinity of SP sources, i.e. near-field probes or metal nanostructures. The field reconstructed in Eq.(4) corresponds to the far-field emission of the near-field SP waves arising from interactions between SPs and objects. Fig. 3a shows typical snapshots from iPM image sequence recorded during the adsorption process of 40-nm Ag nanoparticles onto the Au film in a buffer solution. The amplitude (Fig. 3b) and phase (Fig.3c) images of SP fields scattered by one or more nanoparticles were reconstructed. For comparison, iPM images of artificial particles were simulated, and reconstruction was performed (Fig.S3, supplementary materials). The simulated amplitude and phase images show similar 'ray' and 'spot' patterns in the case of multiple scatterers.

We further examined the splitting of SPs by Au nanowires on the Au surface. Fig. 4a shows the experimental (upper) and simulated (lower) iPM image of single $\mathrm{Au}$ nanowire of about $24.5 \mu \mathrm{m}$ in length and $300 \mathrm{~nm}$ in diameter recorded in air, with the white arrow indicates the propagating direction of SPs. The reconstructed amplitude image of SP fields (Fig. 4b) suggests the splitting capability of the nanowire, with strong SP fields propagating in both longitudinal and orthogonal directions. And the stripe patterns in the reconstructed phase image (Fig. 4c and d) clearly show that the splitted SPs are planar propagating waves.

\section{Improving the spatial resolution}

Propagation of plasmonic waves on metal film degrades the spatial resolution, i.e. the tail-shape patterns in Fig.3. Under the single-scattering condition(36), the object 
waves arising from the interactions between SPs and complex structures with refractive index distribution $o$ could be given by

$$
O=\left(o \cdot E_{s p}\right) * h
$$

where $h$ is the SP fields scattered from a single scatter, which be obtained numerically from Eq.(2) or experimentally from a nanoparticle (Fig.2). Using a 2D-deconvolution algorithm with $h$ as the complex point spread function (PSF), refractive index map of the objects could be quantitatively deduced. Simulation results with random scatterers showed the quantitative measurement of the amplitude and phase in scattering coefficients of the sample (Fig. 5). Note that due to background noise and the difficulties in phase unwrapping, the particles in reconstructed phase images do not appears as well-resolved spots, but the phase values at particle locations correlate well to the preset initial phase. Reconstructed experimental particle images in Fig.3 also showed improved spatial resolution (Fig.S4, supplementary materials).

\section{Discussions}

In this work, we have demonstrated the amplitude- and phase-contrast imaging capability by numerical reconstruction to extend the applications of plasmonic microscopy. Since only one plasmonic image is required for the reconstruction process, the imaging rate depends only on the acquisition speed. In a most-commonly-used setup, the full field-of-view is around $66 \mu \mathrm{m} \times 66 \mu \mathrm{m}$ recorded at 100 frames per second, but could be increased to a higher rate by using a 
high-speed camera. The holographical reconstruction algorithm is dependent on the isolation of the two rings in angular spectrum, which induces noise when the two rings overlap. More sophisticated holographical reconstruction algorithms are readily applicable to reconstruct phase images(37-39), when recording iPM images at an incident angle higher than resonance angle to separate twin images is not practical.

Quantifying phase provides access to refractive index information, which enables the identifications of nanoparticles with different materials. Since the interference patterns are highly dependent on experimental conditions such as focal planes and incident angles, phase quantification by fitting the patterns with numerical simulations thus requires precise control of experimental conditions. Also, it requires the nanoparticle patterns to be well separated from each other and analyzed one by one. In the proposed numerical reconstruction methodology, these issues could be compensated since the incident angle could be estimated, and the images could be reconstructed at different imaging planes. Overlapping between nanoparticle patterns will not affect the reconstruction, and the reconstruction is processed to the full image instead of particle by particle.

As far-field microscopy, the SPs amplitude and phase image reconstructed from iPM is diffraction limited. However, super-resolution information is reserved in the iPM image, since high-frequency components of $\alpha$ were modulated by the propagating SP wave, as shown in Eq. (5). Fig. 6a and b shows the simulation results using two artificial scatterers $260 \mathrm{~nm}$ apart in the direction along or perpendicular to the SP propagating direction, respectively (black curves). Theoretical diffraction-limit 
resolution is about $280 \mathrm{~nm}$ at a wavelength of $680 \mathrm{~nm}$ with a numerical aperture (NA) of 1.49, and the two scatterers are not resolvable directly (left insets and blue curves). Diffraction-limited iPM images were simulated with Eqs. (1) and (3) by further removing the high-frequency components in k-space, reconstructed to obtain full object waves, and deconvoluted to retrieve the refractive index image (right insets and red curves). Obviously, the spatial resolution in the SP propagating direction increases, while in the orthogonal direction resolution reduces due to the information loss through the Au/glass. Therefore, integration with super-resolution techniques such as synthetic aperture microscopy(40) by changing the SPs propagating direction would potentially increase the spatial resolution for super-resolution imaging.

To conclude, we have demonstrated the interferometric plasmonic microscopy as far-field common-path holography for both amplitude and phase- contrast imaging. A planar propagating plasmonic wave was stimulated on a smooth metallic thin film to illuminate the object, and the interference between the leakage radiation through metal/glass structure and the reflective light formed a hologram. Digitally decoding the hologram in angular spectrum gave direct access to the amplitude and phase distribution of plamonic fields. The benefits of proposed methodology include large field-of-view and high imaging rate, no nanoscopic probe or nanostructure, and compatibility for biosensing. While these initial results demonstrate a proof-of-concept for common-path plasmonic holography, various applications are anticipated in bio-nanoparticle analysis and characterizing nanoplasmonic devices.

\section{Materials and Methods}


Microscope system. The iPM system was built on an inverted microscope (IX-83, Olympus, Japan) equipted with a high-numerical-aperture $100 \times$ oil-immersion objective (NA=1.49). The metal/glass chips were BK7 (VWR Corporation, Radnor, PA) glass coverslips coated with $2 \mathrm{~nm}$ chromium and then $47 \mathrm{~nm}$ gold by evaporation. Incident light from a $680 \mathrm{~nm} 1 \mathrm{~mW}$ super luminescence diode (QPhotonics, MI, USA) was refocused and collimated, and illuminated the gold chip in Kretschmann configuration. A CMOS camera (Prime TM; Photometrics, AZ, USA) was used with the system for image recording. Images were acquired at 1024*1024 pixels (full field of view: $66.5 * 66.5 \mu^{2}$ ) and a frame rate of 100 fps. A motorized XY stage (Ludl Electronic Products, Ltd., Hawthorne, NY) was incorporated on the microscope to translate the sensor chip. The incident angle was adjusted by a motorized translational stage (Thorlabs, Newton, NJ).

Nanoparticle and nanowire imaging. Incident angle was scanned to verify the resonance angle, and then fixed at about 73.5 degree for nanoparticle imaging in solution, and about 44.5 degree for nanowire imaging in air. 40-nm Ag nanoparticles were purchased from Aladdin (Shanghai, China), and diluted with double distilled water at 1:1000 v/v. A homemade PDMS solution holder was placed on the Au/glass chip, and filled with $100 \mu \mathrm{L} 1 \times$ PBS buffer. $20 \mu \mathrm{L}$ of the diluted Au-nanoparticle solution was pipetted into the PBS solution, and iPM images are recorded immediately for 2 minutes.

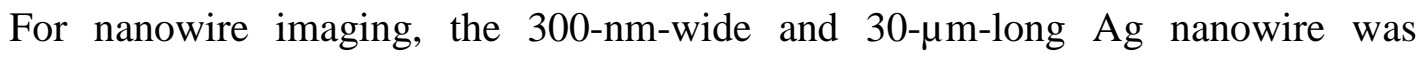
purchased from Aladdin (Shanghai, China), and diluted with ethanol at 1:1000 v/v. A 
2- $\mu \mathrm{L}$ droplet was dried on a clean Au film, leaving randomly positioned nanowires. The chip was rinsed with double distilled water, and blown dry with nitrogen gas. iPM image without nanowires in the field of view was first recorded as the constant background image. Target nanowires were then addressed across the Au surface by laterally translating the stage, and iPM images were recorded accordingly. The background image was then subtracted from the nanowire images to improve the contrast and remove constant reference light in Image J software.

Image processing and simulation. The image reconstruction was carried out using self-developed MATLAB codes. For simulation, we use dielectric constant of $\mathrm{Au}$ $(-11.5477+1.2821 i)$, refractive index of BK-7 glass $(1.515)$, refractive index of water (1.313), and incident angle at 73.5 degree in water and 44.5 degree in air at the wavelength of $680 \mathrm{~nm}$. Angular spectrum reconstruction of the hologram was performed using 2D-FFT.

\section{Supplementary Materials}

Fig.S1 Illustration of wavevector estimation in iPM angular spectrum.

Fig.S2 Simulation results of single nanoparticle image.

Fig.S3 Simulated amplitude and phase images of SP fields scattered by nanoparticles.

Fig.S4 Reconstructed Ag-nanoparticle amplitude- and phase- image.

\section{References and Notes}

1. J. N. Anker, W. P. Hall, O. Lyandres, N. C. Shah, J. Zhao, R. P. Van Duyne, in Nanoscience And Technology: A Collection of Reviews from Nature Journals. 
(World Scientific, 2010), pp. 308-319.

2. G. Baffou, R. Quidant, Nanoplasmonics for chemistry. Chemical Society Reviews 43, 3898-3907 (2014).

3. A. G. Brolo, Plasmonics for future biosensors. Nature Photonics 6, 709 (2012).

4. O. Ekmel, Plasmonics: merging photonics and electronics at nanoscale dimensions. Science 311, 189-193 (2006).

5. M. L. Juan, M. Righini, R. Quidant, Plasmon nano-optical tweezers. Nature photonics 5, 349 (2011).

6. S. Kawata, Y. Inouye, P. Verma, Plasmonics for near-field nano-imaging and superlensing. Nature Photonics 3, 388 (2009).

7. J. Mejía-Salazar, O. N. Oliveira Jr, Plasmonic Biosensing: Focus Review. Chemical reviews 118, 10617-10625 (2018).

8. F. Wei, D. Lu, H. Shen, W. Wan, J. L. Ponsetto, E. Huang, Z. Liu, Wide field super-resolution surface imaging through plasmonic structured illumination microscopy. Nano letters 14, 4634-4639 (2014).

9. W. Wang, Y. Yang, S. Wang, V. J. Nagaraj, Q. Liu, J. Wu, N. Tao, Label-free measuring and mapping of binding kinetics of membrane proteins in single living cells. Nature chemistry 4, 846 (2012).

10. S. Wang, X. Shan, U. Patel, X. Huang, J. Lu, J. Li, N. Tao, Label-free imaging, detection, and mass measurement of single viruses by surface plasmon resonance. Proceedings of the National Academy of Sciences 107, 16028-16032 (2010).

11. Y. Yang, H. Yu, X. Shan, W. Wang, X. Liu, S. Wang, N. Tao, Label - Free Tracking of Single Organelle Transportation in Cells with Nanometer Precision Using a Plasmonic Imaging Technique. Small 11, 2878-2884 (2015).

12. H. Yu, X. Shan, S. Wang, H. Chen, N. Tao, Plasmonic imaging and detection of single DNA molecules. ACS nano 8, 3427-3433 (2014).

13. Y. Yang, G. Shen, H. Wang, H. Li, T. Zhang, N. Tao, X. Ding, H. Yu, Interferometric plasmonic imaging and detection of single exosomes. Proceedings of the National Academy of Sciences 115, 10275-10280 (2018).

14. Y. Jiang, H. Su, W. Wei, Y. Wang, H.-Y. Chen, W. Wang, Tracking the rotation of single CdS nanorods during photocatalysis with surface plasmon resonance microscopy. Proceedings of the National Academy of Sciences, 201820114 (2019).

15. K. Syal, R. Iriya, Y. Yang, H. Yu, S. Wang, S. E. Haydel, H.-Y. Chen, N. Tao, Antimicrobial Susceptibility Test with Plasmonic Imaging and Tracking of Single Bacterial Motions on Nanometer Scale. ACS nano 10, 845-852 (2015).

16. C. Qian, G. Wu, D. Jiang, X. Zhao, H. B. Chen, Y. Yang, X. W. Liu, Identification of Nanoparticles via Plasmonic Scattering Interferometry. Angewandte Chemie International Edition 58, 4217-4220 (2019).

17. S. Wu, H. Ho, W. Law, C. Lin, S. Kong, Highly sensitive differential phase-sensitive surface plasmon resonance biosensor based on the MachZehnder configuration. Optics Letters 29, 2378-2380 (2004). 
18. A. V. Kabashin, S. Patskovsky, A. N. Grigorenko, Phase and amplitude sensitivities in surface plasmon resonance bio and chemical sensing. Optics Express 17, 21191-21204 (2009).

19. A. G. Notcovich, V. Zhuk, S. Lipson, Surface plasmon resonance phase imaging. Applied Physics Letters 76, 1665-1667 (2000).

20. H. Raether, in Surface plasmons on smooth and rough surfaces and on gratings. (Springer, 1988), pp. 4-39.

21. C. E. Berger, R. P. Kooyman, J. Greve, Resolution in surface plasmon microscopy. Review of scientific instruments 65, 2829-2836 (1994).

22. F. A. Banville, T. Söllradl, P.-J. Zermatten, M. Grandbois, P. G. Charette, Improved resolution in SPR and MCWG microscopy by combining images acquired with distinct mode propagation directions. Optics letters 40, 1165-1168 (2015).

23. Y. Kuai, J. Chen, X. Tang, Y. Xiang, F. Lu, C. Kuang, L. Xu, W. Shen, J. Cheng, H. Gui, Label-free surface-sensitive photonic microscopy with high spatial resolution using azimuthal rotation illumination. Science Advances 5, eaav5335 (2019).

24. T. Son, C. Lee, J. Seo, I.-H. Choi, D. Kim, Surface plasmon microscopy by spatial light switching for label-free imaging with enhanced resolution. Optics letters 43, 959-962 (2018).

25. H. Yu, X. Shan, S. Wang, H. Chen, N. Tao, Molecular scale origin of surface plasmon resonance biosensors. Analytical chemistry 86, 8992-8997 (2014).

26. H. Yu, X. Shan, S. Wang, N. Tao, Achieving high spatial resolution surface plasmon resonance microscopy with image reconstruction. Analytical Chemistry 89, 2704-2707 (2017).

27. Y. Jiang, W. Wang, Point Spread Function of Objective-Based Surface Plasmon Resonance Microscopy. Analytical Chemistry 90, 9650-9656 (2018).

28. A. Drezet, C. Genet, Imaging surface plasmons: from leaky waves to far-field radiation. Physical review letters 110, 213901 (2013).

29. S. I. Bozhevolnyi, B. Vohnsen, Near-field optical holography. Physical review letters 77, 3351 (1996).

30. J. W. Nelson, G. R. Knefelkamp, A. G. Brolo, N. C. Lindquist, Digital plasmonic holography. Light: Science \& Applications 7, 52 (2018).

31. P. Dvořák, M. Kvapil, P. Bouchal, Z. Édes, T. Šamořil, M. Hrtoň, F. Ligmajer, V. Křápek, T. Šikola, Near-field digital holography: a tool for plasmon phase imaging. Nanoscale 10, 21363-21368 (2018).

32. N. Rotenberg, L. Kuipers, Mapping nanoscale light fields. Nature Photonics 8, 919 (2014).

33. C. Zhao, Y. Liu, Y. Zhao, N. Fang, T. J. Huang, A reconfigurable plasmofluidic lens. Nature communications 4, 2305 (2013).

34. E. Descrovi, E. Barakat, A. Angelini, P. Munzert, N. De Leo, L. Boarino, F. Giorgis, H. P. Herzig, Leakage radiation interference microscopy. Optics letters 38, 3374-3376 (2013).

35. A. Drezet, A. Hohenau, D. Koller, A. Stepanov, H. Ditlbacher, B. Steinberger, 
F. Aussenegg, A. Leitner, J. Krenn, Leakage radiation microscopy of surface plasmon polaritons. Materials science and engineering: B 149, 220-229 (2008).

36. S. I. Bozhevolnyi, V. Coello, Elastic scattering of surface plasmon polaritons: modeling and experiment. Physical Review B 58, 10899 (1998).

37. G. Zheng, R. Horstmeyer, C. Yang, Wide-field, high-resolution Fourier ptychographic microscopy. Nature photonics 7, 739 (2013).

38. M. Liebling, T. Blu, M. Unser, Complex-wave retrieval from a single off-axis hologram. JOSA A 21, 367-377 (2004).

39. U. Schnars, W. P. Jüptner, Digital recording and numerical reconstruction of holograms. Measurement science and technology 13, R85 (2002).

40. T. S. Ralston, D. L. Marks, P. S. Carney, S. A. Boppart, Interferometric synthetic aperture microscopy. Nature Physics 3, 129 (2007).

\section{Acknowledgments}

We acknowledge financial support from the National Natural Science Foundation of China (\#61805136) and Shanghai Pujiang Program (\#18PJ1406400). H.Y. conceived the study. Y.Y. performed the experiments. Y.Y. and H.Y. developed the algorithms. C.Z. and A.L.K. helped with the sample preparation and experiments. Y.Y. and H.Y. wrote the paper. The authors declare no competing financial interest. 
Figures.

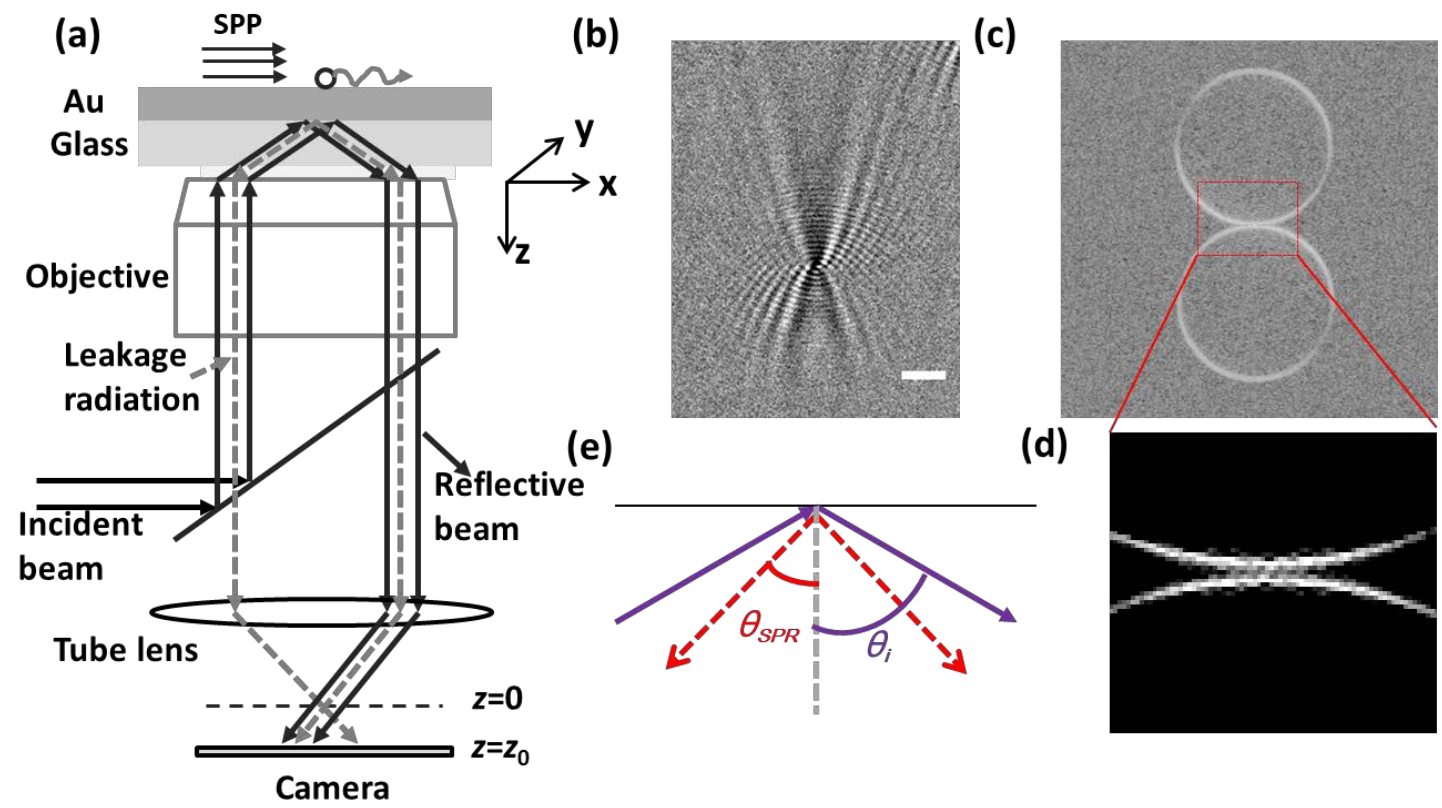

Fig. 1 Mechanism of amplitude- and phase-contrast plasmonic microscopy. (a)

Schematics. (b) iPM image of a 40-nm Ag nanoparticle in real space and (c) in k-space. (d) Zoomed-in image of (c). (e) Illustration of incident angle and resonance angle. 
(a)

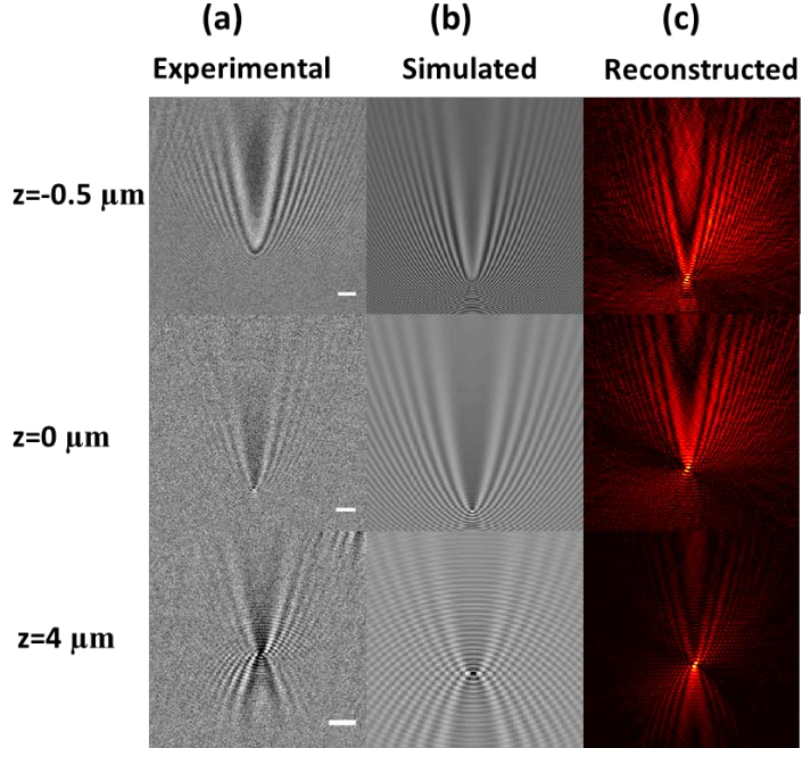

Fig. 2 iPM images of 40-nm Ag nanoparticles at different focal planes. (a) Experimental, (b) simulated, (c) reconstructed. Scalebars: $2 \mu \mathrm{m}$. 


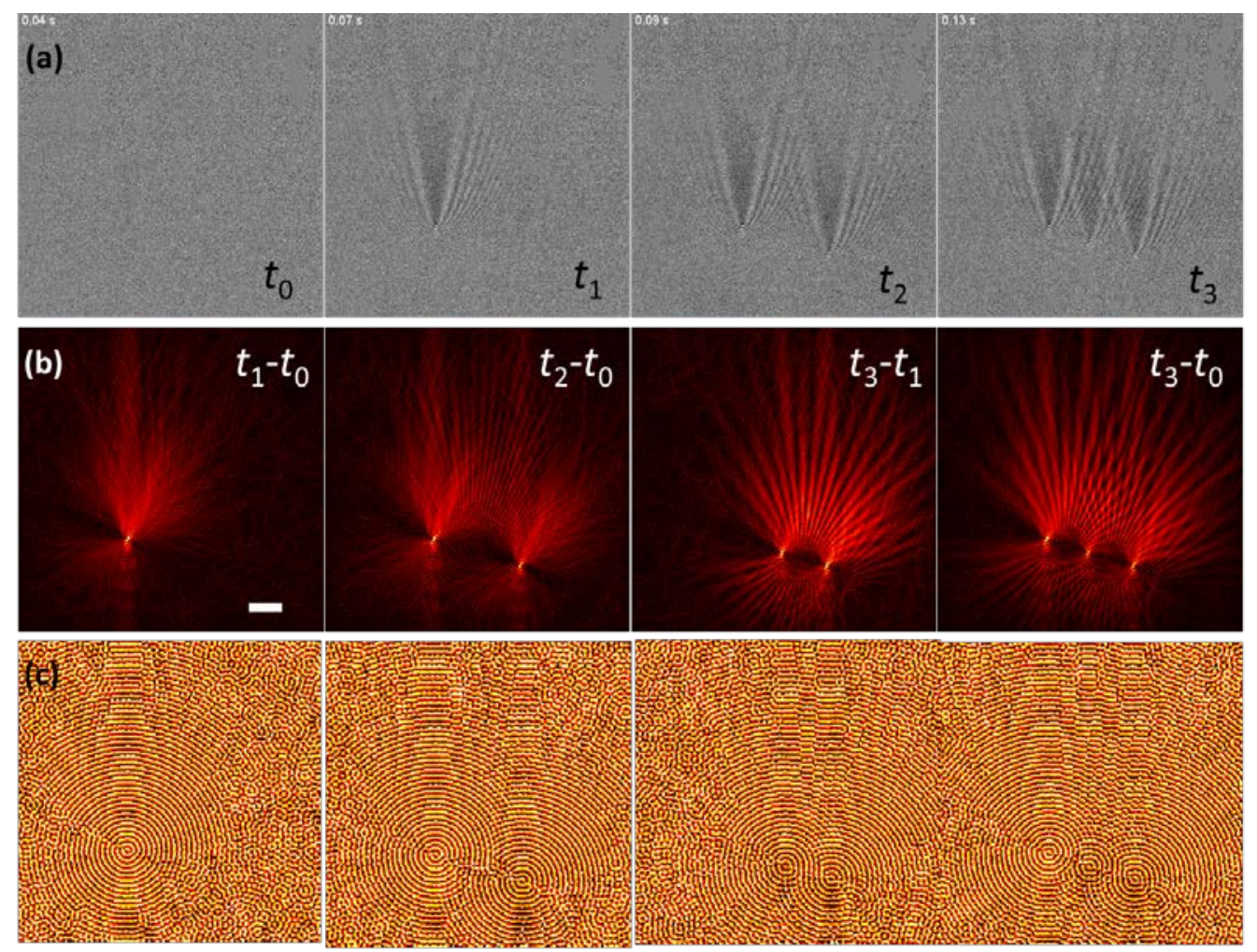

Fig. 3 Imaging SP fields scattered by 40-nm Ag nanoparticles. (a) Snapshots of iPM images during the nanoparticle binding process. (b) Amplitude and (c) phase images of SP fields scattered by Ag nanoparticles. Scalebars: $4 \mu \mathrm{m}$. 

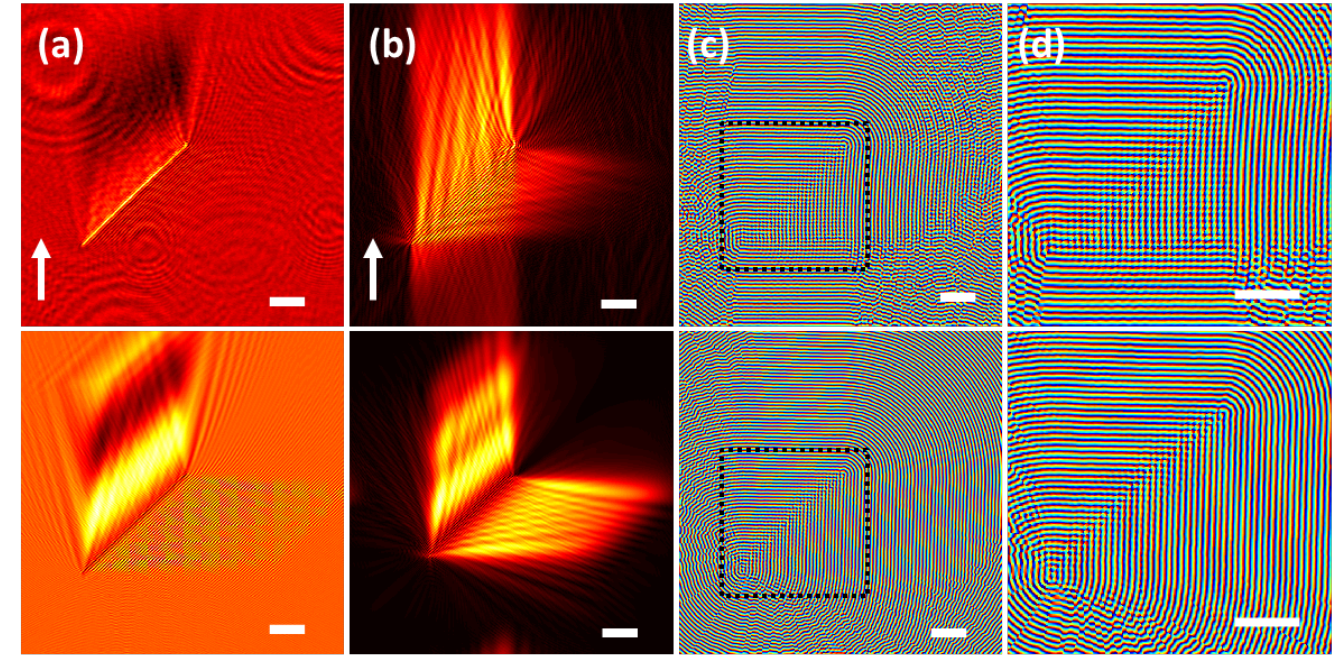

Fig. 4 Imaging SPs beam splitting by Au nanowires. Experimental (upper) and theoretical (lower) (a) iPM images, (b) amplitude and (c) phase images of SP fields from interaction with Au nanowire, and (d) zoomed-in images of the square region in (c). Scalebars: $5 \mu \mathrm{m}$. 

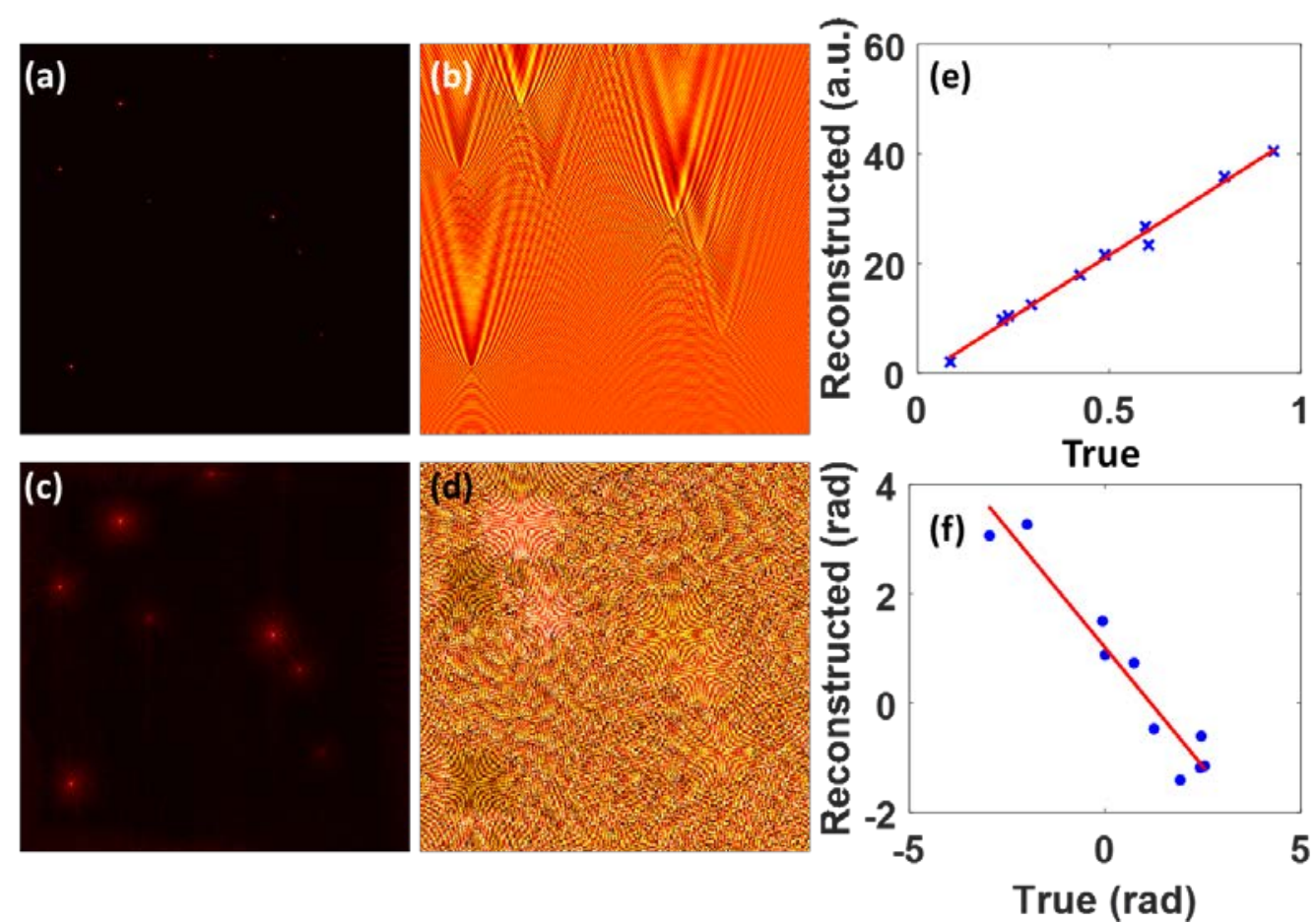

Fig.5 Simulation of amplitude and phase quantification. (a) Simulated point scatterer at random position with random scattering coefficient and initial phase change. (b) Simulated iPM holograms. (c) Reconstructed amplitude and (d) phase imagesc. (e) Comparison of reconstructed and real amplitude and (f) phase values at the object location. 

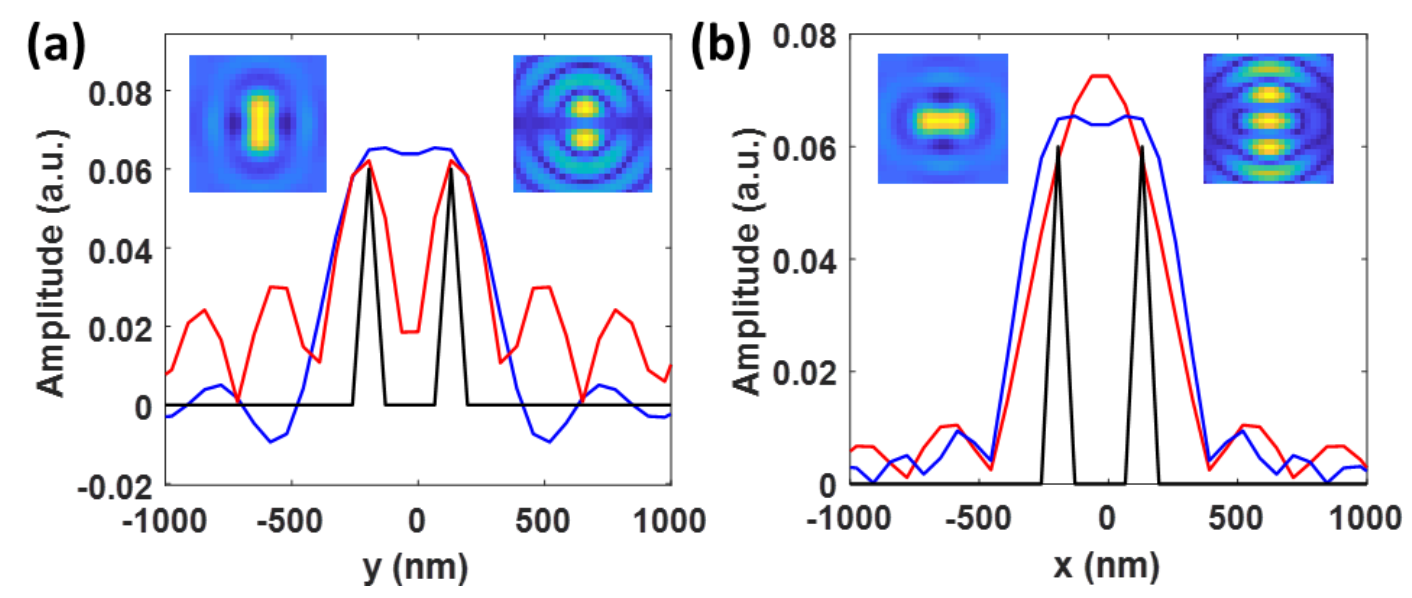

Fig.6 Super-resolution imaging. Line profiles of diffraction-limited optical images (blue, left insets), reconstructed refractive index amplitude image (red, right insets), and true amplitude of two point scatterers (dark) with $260 \mathrm{~nm}$ apart in (a) y- and (b) xaxis. 
Quantitative amplitude- and phase- contrast plasmonic mic... (1.29 MiB) view on ChemRxiv • download file 


\section{Supplementary materials}

\section{Quantitative amplitude- and phase- contrast plasmonic microscopy with high spatial resolution}

Yuting Yang, Chunhui Zhai, Ab Lateef Khan, Hui Yu*

Institute for Personalized Medicine, School of Biomedical Engineering, Shanghai Jiao

Tong University, Shanghai, 200030, China

Correspondence should be sent to:

Prof. Hui Yu

Email: hui.yu@sjtu.edu.cn

Tel/Fax: +86-21-62933948 


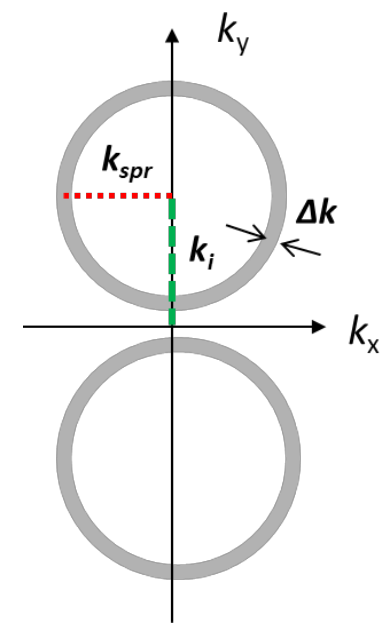

Fig. S1 Illustration of wavevector estimation in iPM angular spectrum. 


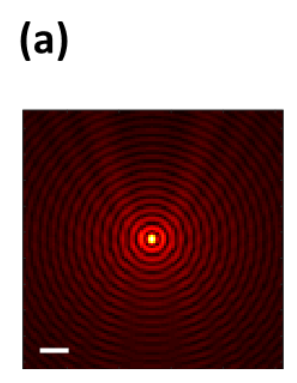

(b)

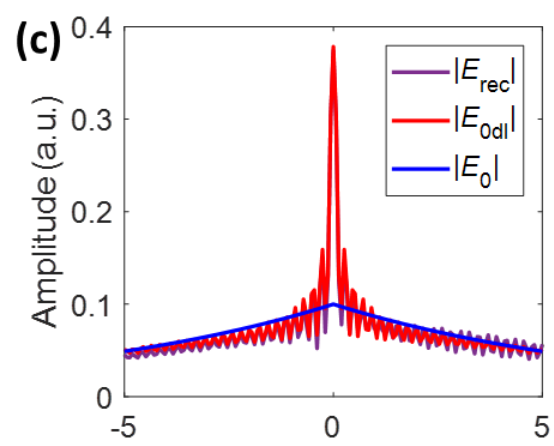

(d)
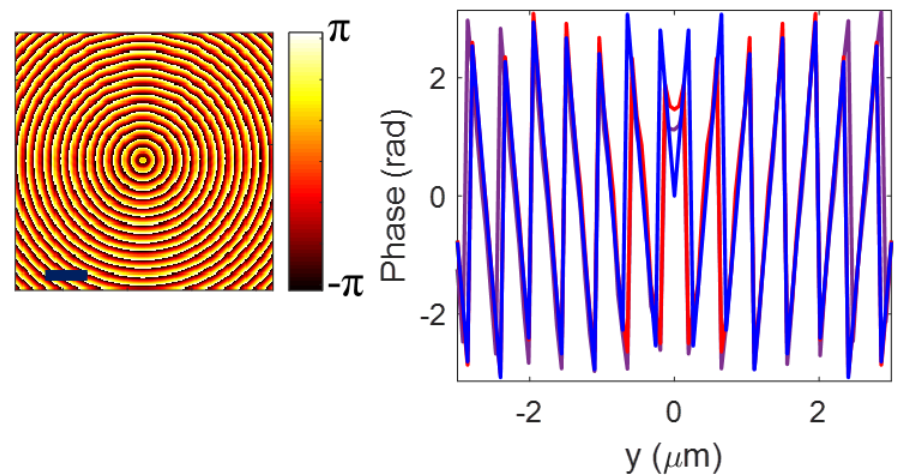

Fig.S2 Simulation results of single nanoparticle image. (a) (c) Amplitude and (b) (d) phase images and profiles of nanoparticle images without (blue) and with (red) pupil function, and reconstructed (purple). 


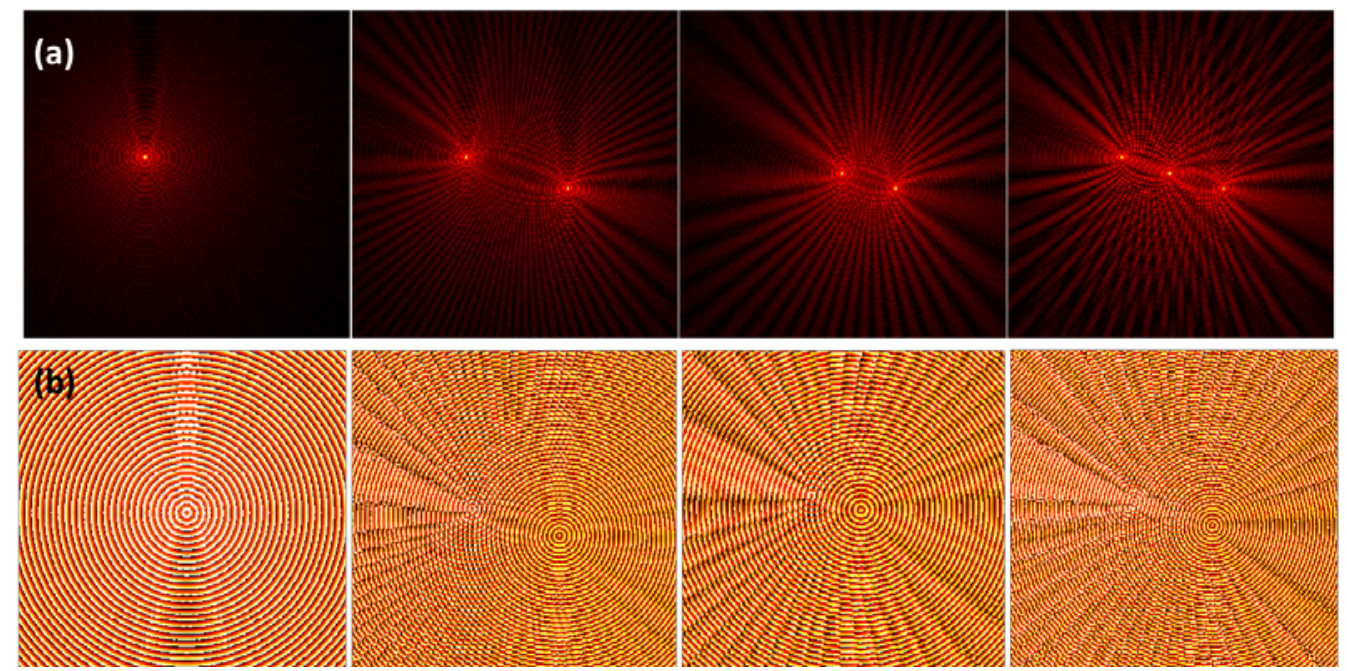

Fig. S3 Simulated amplitude (upper) and phase (lower) images of SP fields scattered by nanoparticles. (a) Single particle, (b) and (c) two particles, (d) Three particles. 


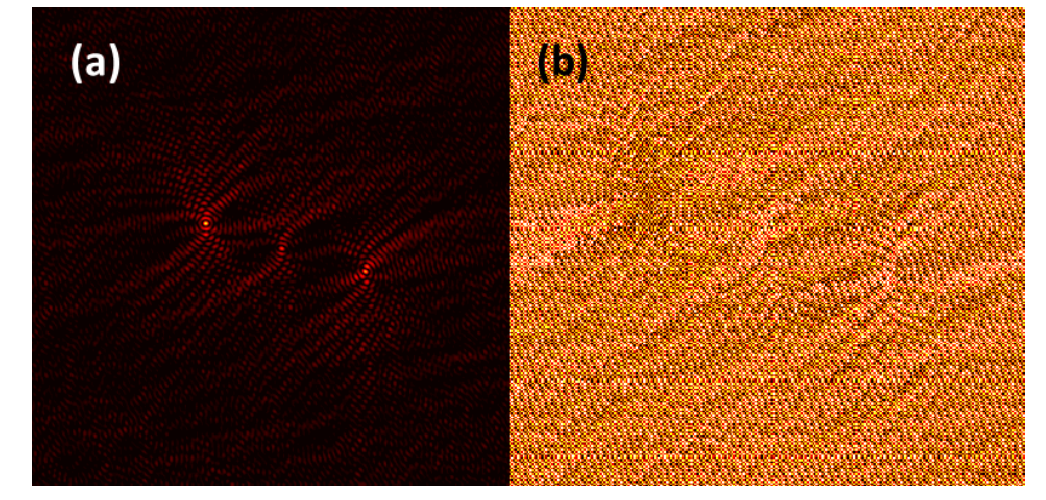

Fig.S4 Reconstructed Ag-nanoparticle (a) amplitude- and (b) phase- image. 
